\title{
The Keck Cosmic Web Imager
}

\author{
Chris Martin a , Anna Moore ${ }^{\mathrm{a}}$, Patrick Morrissey ${ }^{\mathrm{a}}$, Matt Matuszewski ${ }^{\mathrm{a}}$, Shahin Rahman ${ }^{\mathrm{a}}$, Sean \\ Adkins ${ }^{\mathrm{b}}$, Harland $\mathrm{Epps}^{\mathrm{c}}$ \\ ${ }^{a}$ California Institute of Technology, Cahill Center for Astrophysics, 1216 E. California Blvd., \\ Pasadena, CA 91125 USA \\ ${ }^{\mathrm{b}}$ W. M. Keck Observatory, 65-1120 Mamalahoa Highway, Kamuela, HI, USA 96743 \\ ${ }^{\mathrm{c}}$ UCO/Lick Observatory, 1156 High St., Santa Cruz, CA, USA 95062
}

\begin{abstract}
We are designing the Keck Cosmic Web Imager (KCWI) as a new facility instrument for the Keck II telescope at the W. M. Keck Observatory (WMKO). KCWI is based on the Cosmic Web Imager (CWI), an instrument that has recently had first light at the Hale Telescope. KCWI is a wide-field integral-field spectrograph (IFS) optimized for precision sky limited spectroscopy of low surface brightness phenomena. KCWI will feature high throughput, and flexibility in field of view (FOV), spatial sampling, bandpass, and spectral resolution. KCWI will provide full wavelength coverage $(0.35$ to $1.05 \mu \mathrm{m})$ using optimized blue and red channels. KCWI will provide a unique and complementary capability at WMKO (optical band integral field spectroscopy) that is directly connected to one of the Observatory's strategic goals (faint object, high precision spectroscopy), at a modest cost and on a competitive time scale, made possible by its simple concept and the prior demonstration of CWI.
\end{abstract}

Keywords: Instrumentation, Integral Field Spectroscopy, Visible

\section{INTRODUCTION}

The Keck Cosmic Web Imager (KCWI) is a wide field, seeing limited, integral field spectrograph (IFS) optimized for precision sky limited spectroscopy of low surface brightness phenomena. KCWI will feature high throughput and flexibility in field of view (FOV), spatial sampling, bandpass, and spectral resolution. KCWI will provide full wavelength coverage $(0.35$ to $1.05 \mu \mathrm{m})$ using optimized blue and red channels. The preliminary design phase of KCWI has recently been initiated through collaboration between CIT, UCSC, and WMKO.

KCWI is based on the Cosmic Web Imager (CWI) recently commissioned at the Palomar Observatory ${ }^{[1]}$. CWI is a single channel instrument with a fixed spatial sampling scale and a single adjustable VPH grating and an articulated camera with $2 \mathrm{k} \mathrm{x} 4 \mathrm{k}$ mid-band optimized CCD. KCWI will build on the heritage of CWI with a two channel design offering three selectable spatial sampling scales and a selection of VPH gratings. KCWI will be located at the right Nasmyth focus of the Keck II telescope. A plan view of an initial concept for the opto-mechanical layout of KCWI is shown in Figure 4 .

\section{SCIENCE}

\subsection{Science goals}

KCWI is designed to provide visible band, integral field spectroscopy (IFS) at WMKO with moderate to high spectral resolution. KCWI will benefit from the excellent seeing, low sky background, and large aperture, providing a worldleading capability. KCWI will provide a number of substantial benefits, including flexible image resolution and binning, flexible spectral resolution, excellent sky subtraction, and the highest possible throughput optimized for both the blue and red ends of the visible wavelength range.

KCWI will address a broad range of science applications, a number of which are highlighted in this section.

(1) Young Stars and Jets. KCWI will probe the kinematics and ionization physics of young stellar jets (such as HerbigHaro objects) with high resolution that can resolve the $<50 \mathrm{~km} / \mathrm{s}$ line widths. With its excellent sensitivity KCWI will be suited to low surface brightness targets and can search for faint shocks and relic signatures of prior outflow history.

Ground-based and Airborne Instrumentation for Astronomy III, edited by lan S. McLean,

Suzanne K. Ramsay, Hideki Takami, Proc. of SPIE Vol. 7735, 77350M · C 2010

SPIE $\cdot$ CCC code: $0277-786 \times / 10 / \$ 18 \cdot$ doi: $10.1117 / 12.858227$ 
(2) Evolved Star Nebulae offer a wide variety of physical phenomena which can be highly informative about poorlymodeled mass-loss processes and the physics of outflows. Pulsar wind nebulae are excellent candidates for 2D kinematic, ionization, and density/pressure mapping. These nebula are now believed to be strong ultra-high-energy gamma-ray sources. Asymptotic Giant Branch stars in the thermal pulse phase show copious mass loss episodes. But many unusual nebulae are extremely faint in the optical ${ }^{[2],[3]}$ and show complex morphology, requiring 2D spectroscopic imaging with high sensitivity.

(3) Light Echoes in the Milky Way and nearby galaxies are historical evidence for supernovae and possibly other energetic explosions. Extended, low surface brightness structures mapped with KCWI could provide spectroscopic evidence confirming their SNe or GRB origin and object class.

(4) Star Clusters. There has been tantalizing evidence for intermediate mass black holes at the center of several globular clusters $^{[4]}$. KCWI would provide high quality $2 \mathrm{D}$ kinematics with $15 \mathrm{~km} / \mathrm{s}$ resolution along with abundance mapping in old and young clusters.

(5) Low Surface Brightness Galaxies and Star Formation in Extreme Regimes. An exciting area of research is the study of low surface brightness, low mass density galaxies and associated star formation. Topics include extending star formation laws to the lowest densities and galaxy masses, testing the universality of the initial mass function in the lowdensity regime, studying the stellar populations and searching for direct evidence of feedback. Low mass galaxies are often dark matter dominated, and provide a unique opportunity to probe the distribution of dark matter in galaxy cores and test dark matter models. A long-standing problem for these models that can be investigated is the lack of the "cusps" predicted in galaxy cores if the dark matter is cold and not self-interacting.

(6) Galactic Halos, Thick Disks, Streams, Intracluster Light. Recently, deep photometry has produced spectacular results showing that galaxies very often host thick disks, large diffuse halos, complex tidal streams and other extended features. These features are believed to be relics of prior minor and major mergers and interactions with satellites. The more extended structures have cosmological coherence timescales and KCWI offers the possibility of attempting kinematic, stellar population, and even abundance measurements of these highly extended low surface brightness structures. Spectroscopic analysis combined with modeling could provide strong constraints on merger histories and their impact on galaxy evolution. Intracluster light has been detected in many nearby clusters, and is believed to be produced by stars stripped from their galaxies as the groups and cluster assemble, as well as by stars formed during interactions in tidal streams. KCWI would provide kinematic maps and line-index diagnostics that could yield stellar age and metallicity information crucial to unraveling these stellar relics.

(7) AGN/QSO/Galaxy Co-evolution. One of the most interesting and controversial issues in galaxy evolution theory is the role of AGN feedback on determining the star formation history of galaxies and the black hole mass/stellar velocity dispersion relationship. One approach to this is to study nearby galaxies with AGN and extended emission to understand the physical properties, nature of ionization, mechanical energy flux, and ultimately the power deposited in the ISM to determine the effectiveness of feedback for self-regulation.

(8) Circum-QSO Medium. Prior to and shortly after QSOs are born, the Circum-QSO medium (CQM) may be a fair representation of the typical QSO environment. In particular, the CQM, illuminated by the QSO could provide a very useful picture of the CGM around galaxies at $2<\mathrm{z}<6$ through high signal to noise ratio (SNR) emission maps. KCWI's simple high throughput optical design should allow observations with a high dynamic range. Coupled with an occulting spot, it should be possible to explore the QSO host galaxy, host environment, and intervening absorber galaxy properties.

\section{(9) Low and High Redshift Galaxy Structure}

- Galaxy stellar kinematics and populations: Bulges. KCWI would provide exquisite maps of stellar kinematics and populations traced by the usual absorption line indices in the high surface brightness cores of typical bulge systems, and thus furnish high order kinematic moments and spatial mapping which could separate multiple structural components of bulges assembled over cosmological time. KCWI could extend kinematic and stellar population measurements to the unexplored low surface brightness regions in the outskirts of galaxies, probing relic structures with long dynamical times and memories of their assembly history. In low surface brightness dwarf galaxies, kinematic maps will provide constraints on dark matter distribution and properties, allowing an attempt to address the "cusp problem". 
- Galaxy stellar kinematics and populations: Star-forming galaxies. In star forming galaxies it may be possible to map age vs. position in galaxies and reconstruct the local and global star formation history, and relate these to causal factors such as gas density, dynamical instabilities, ISM turbulence, and global star formation laws. KCWI extends these observations to low surface brightness irregulars, providing maps of complex star forming regions that could test for the impact of feedback, ionization, and turbulent regulation on the history of star formation in these low mass systems.

- High z galaxy environments / assembly. High redshift galaxies are likely to lie in complex, multi-component systems with fainter companions and extended stellar and gaseous features. KCWI will detect these fainter companions and measure relative velocities and other properties (e.g., star formation rate). $2 \mathrm{D}$ coverage permits summing light from extended components and producing the highest resolution spectra with the highest possible SNR.

- Strong lens systems are complex, 2D systems that could be analyzed in detail using KCWI spectral images, providing information about the lensing galaxy (redshift, stellar velocity dispersion, mass, stellar populations) and lensed galaxy (stellar populations, star formation rate, age, metallicity, etc.).

- Galactic feedback at low and high redshift. Feedback from massive star formation is one of the critical missing components in a complete understanding of galaxy formation and evolution. At high redshift, KCWI will probe outflows and their radiative impacts (if any) on the surrounding CGM/IGM (see below). At low redshift, KCWI can be used to map galactic outflows on large scales (more distant galaxies) and in nearby galaxies on the scale of star formation regions. It should be possible to detect very low surface brightness gaseous halos, galactic fountains, and superwind outflows even if the primary energy loss is not detectable.

(10) Circum-Galactic Medium. One of the forefront topics today is the connection between galaxies and the gas in their dark matter halos (we term this the Circum-Galactic Medium or CGM) as well as with the IGM gas in the cosmic web. KCWI is optimal for detecting low surface brightness emission from redshifted Ly $\alpha$ as well as resonance lines of OVI1033 $\AA$, CIV1550 $\AA$ over the redshift range $2<\mathrm{z}(\operatorname{Ly} \alpha)<6$. For example, there have been tentative detections of Ly $\alpha$ halos around Lyman Break Galaxies, which may trace gas in dark matter halos which is either fueling star formation and galaxy formation, or gas that has been energized by galactic superwinds. A simulated observation is shown in Figure 1. Separating the different emission components, isolating the excitation mechanism for resonance line emission (scattering, recombination, shocks), and removing foreground objects and companions associated with the target galaxy requires $2 \mathrm{D}$ spectral imaging with excellent sky subtraction, the highest possible sensitivity, good imaging resolution, and moderate $(\mathrm{R} \sim 3000)$ spectral resolution to separate components at different velocities and to resolve velocity structure produced by bulk motions and radiative transfer in the resonance line.

Figure 1: Simulation of Lya $(\mathrm{z} \sim 2.5)$ emission from Circum-Galactic Medium performed by Greg Bryan, and showing the KCWI FOV

The color scale is logarithmic, with medium green corresponding to the 5 sigma sensitivity limit in $5 \times 5$ arcsecond ${ }^{2}$ emission regions for $K C W T$ after 8 hours of integration (orange is 10 times higher intensity, blue ten times lower). Left: CGM with no feedback, narrow/fine IFU. Also shown is the GMOS IFU assuming I slit mode. Right: CGM with feedback, medium FOV IFU (see Table 2).
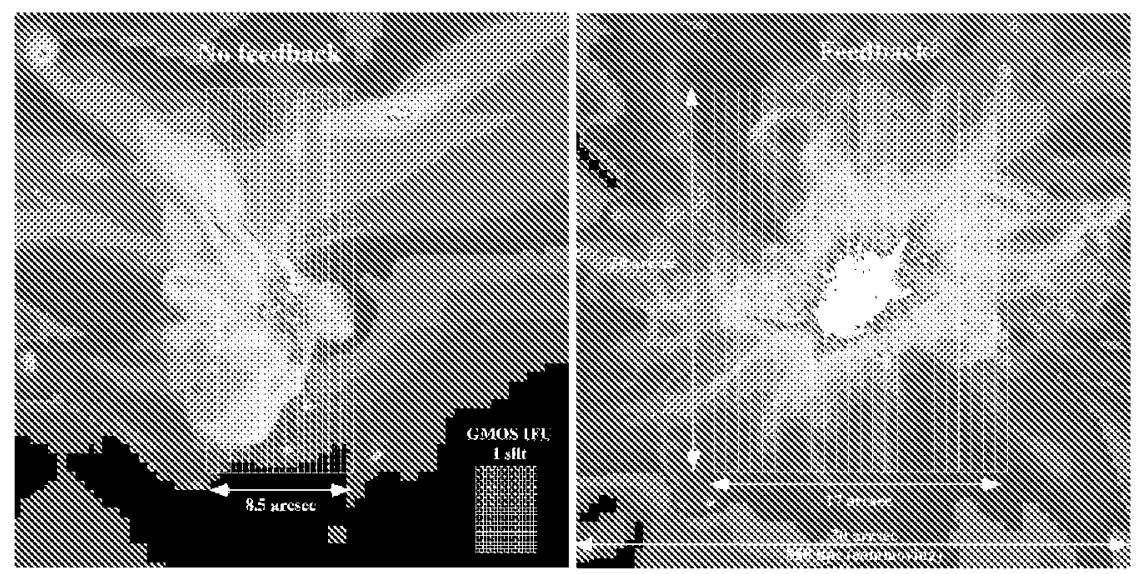

(11) Ly $\alpha$ Emitters and Blobs have been detected using narrow-band imaging but are still poorly understood. 2D spectral mapping will provide kinematic information, constraints on associated metal line emission (OVI, CIV, etc.), and constraints on continuum sources that may be powering the blobs. 


\section{(12) Emission from the IGM/Cosmic Web}

- Metagalactic ionizing background can be measured in principal at high redshift using Lya fluorescence, and at low redshift using $\mathrm{H} \alpha$ or $\mathrm{H} \beta$ emission. Reaching the very low surface brightness required, and separating out other mechanisms for producing the emission is best accomplished with $2 \mathrm{D}$ spectral mapping designed to probe emission strengths $<0.1$ to $1 \%$ of sky.

- Emission from the Cosmic Web may be detectable at the limits of KCWI sensitivity. Figure 2 shows a prediction of Ly $\alpha$ emission at $\mathrm{z} \sim 6$ from filaments of the IGM ${ }^{[5]}$, with the KCWI coarse FOV superimposed as a mosaic of 15 pointings. Emission at the green-yellow level is definitely detectable using statistical filament stacking techniques and may be even detected via direct imaging.

- Detecting and mapping the Cosmic Web in emission will offer a profound new technique for studying the coevolution of galaxies and the IGM and provide a new tool for large-scale structure and cosmological measurement.

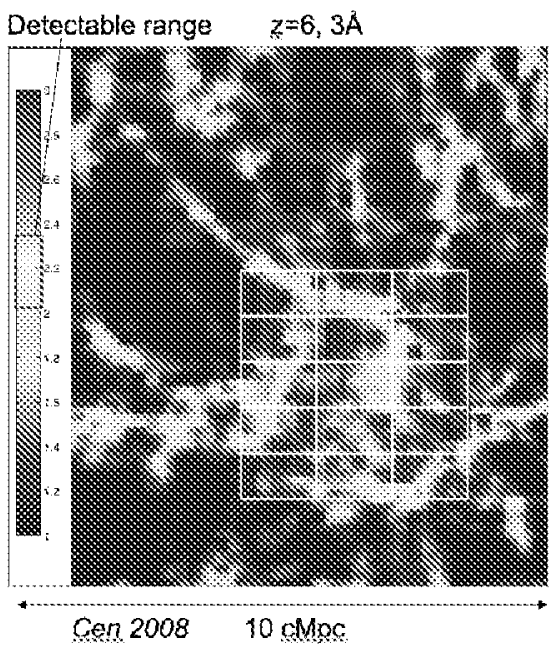

Figure 2: Model of emission from the IGM in Ly $\alpha$ at redshift of $\mathrm{z} \sim 6$

(13) Reionization Bubbles. Models predict ${ }^{[6]}$ that during the epoch of reionization $(z>6)$ growing HII regions should be detectable by extremely faint and highly extended (many arcminutes) Ly $\alpha$ emission, detectable by $\mathrm{KCWI}$ to a redshift of $\mathrm{z} \sim 7.2$. KCWI will be designed to provide excellent sky and point source subtraction and sensitivity to extended emission using the entire FOV to provide the highest SNR. This is a pioneering but challenging observation that cannot be performed by any other facility.

\subsection{Science Requirements}

The science applications in the preceding section were provided by the WMKO observing community in response to a request for science cases for the proposed KCWI instrument. In Table 1 we summarize these science applications and the requirements for key KCWI performance parameters for each application.

The KCWI science requirements were developed by starting with the current capabilities of CWI, which is designed specifically to detect and map emission from the IGM, and therefore is built to obtain high quality sky subtraction and medium spectral resolution $(R=5000)$ in a relatively narrow band. CWI has a single channel and with its baseline high dispersion volume phase holographic (VPH) gratings has a small instantaneous bandpass optimized in the blue. By synthesizing the science objectives in Table 1 into a common set of requirements given in Table 2, KCWI is conceived as a general-purpose integral field spectrograph built upon the CWI design with broad wavelength coverage, flexible slicer spatial sampling and FOV, with flexible bandpass and spectral resolution. Each of the KCWI science requirements is briefly discussed here.

Spatial Resolution. Many observations, usually complex objects with fine spatial structure such as young stars, crowded stellar clusters, AGN and distant galaxies, require the best obtainable spatial resolution, implying Nyquist sampling of the median 0.7 " seeing on Mauna Kea. Observations of more extended structures (light echos in the Milky Way, galaxy halos, intracluster light, IGM, reionization bubbles) require a wider FOV and can accommodate lower spatial resolution as they are often observed in "light bucket" mode, in which all the light from the integral field unit (IFU) is combined to reach the lowest surface brightness and highest SNR possible. The IFU by design samples the 2D field with individual slices, which for extended sources determines the spatial resolution in one dimension (and the spectral resolution), assuming a well-corrected camera design. IFU and camera optical performance and the CCD format determine the spatial resolution along the slices, which for KCWI will always be Nyquist sampled for 0.6 " seeing. KCWI achieves flexible sampling and FOV using a selectable slicer design, discussed below.

FOV. There are a wide range of requirements for field of view, ranging from 5 " to 10" for young stars to a significant portion of an arcminute for nearby galaxies, clusters, blank sky surveys and light bucket observations. KCWI provides 
the maximum possible FOV for a given application by using a selectable image slicer, large format detectors, wide FOV cameras, and high-dispersion gratings.

Spectral Resolution. The requirements for spectral resolution range from $\mathrm{R} \sim 1000$ for measuring SEDs and strong emission lines in faint extended objects and high-z galaxies to $\mathrm{R} \sim 3000$ to 5000 for kinematic mapping to $\mathrm{R} \sim 15,000$ to 20,000 for abundance mapping projects. KCWI accommodates these diverse requirements by a) providing three slicer formats, b) providing slit limited spectral resolution for all three formats, c) multiple grating and dispersion choices, and d) a dual channel design.

Bandpass. A general purpose imaging spectrograph requires sensitivity over as wide a bandpass as possible. IGM, high$\mathrm{z}$ galaxy, and abundance topics require blue sensitivity, while reionization, $\mathrm{z}>5$ galaxy, and moderate- $\mathrm{z} \mathrm{H} \alpha$ mapping require extended red coverage beyond $1 \mu \mathrm{m}$. KCWI maximizes bandpass by using a dual channel design, a blueoptimized camera with a short wavelength cutoff of $3500 \AA$, a red-optimized channel with a fully-depleted CCD that has a $1.05 \mu \mathrm{m}$ cutoff. The lowest dispersion gratings will allow observation of the full 0.35 to $1.05 \mu \mathrm{m}$ band simultaneously at $\mathrm{R} \sim 1000$ to 3600 depending on the slicer chosen. Higher dispersion gratings can be tuned to desired bands. For example, even with the finest slicer and highest dispersion gratings, it is possible to simultaneously cover $\mathrm{H} \beta$ and the [OIII] doublet with the blue channel, and $\mathrm{H} \alpha$, [NII], and [SII] with the red channel, all at $\mathrm{R} \sim 20,000$.

Efficiency. The highest possible efficiency is a core KCWI requirement in order to exploit imaging spectroscopy for exploring the ultra-low surface brightness universe. The requirement is $20 \%$ end-to-end efficiency (including telescope and atmosphere) over 0.4 to $1.0 \mu \mathrm{m}$, with a goal of $25 \%$. It is achieved by using a dual-channel design separately optimized for blue and red, a simple optical design with a small number of surfaces, optimized coatings for the individual channels, state-of-the-art broadband coatings for the pre-dichroic optics, high-efficiency VPH gratings, and state-of-the-art CCDs.

Sky/object subtraction. Many of the highest priority KCWI science objectives target the ultra-low-surface-brightness Universe. For example, measuring reionization bubbles, emission from the CGM and IGM, and determining the physical properties of galaxy halos and streams all push below 0.1 to $1 \%$ of sky. Using KCWI in "light-bucket" mode, that is summing all the spatial samples to obtain a single, very deep spectrum of an extended source, will achieve these required sensitivities only if high-precision sky subtraction is possible. This is illustrated on the left side of Figure 3 which shows an $\mathrm{R}=1000,16$ hour integration using the coarse slicer, with three different levels of sky subtraction precision $\left(\mathrm{f}_{\text {sky }}=10^{-2}\right.$, $10^{-3}, 10^{-4}$ at $1 \sigma$ ). With $1 \%$ accuracy the flux is subtraction-limited to $10^{-18} \mathrm{erg} \mathrm{cm}^{-2} \mathrm{~s}^{-1}$ arcseconds ${ }^{-2}$. With $0.01 \%$ accuracy this drops to $\sim 2 \times 10^{-20} \mathrm{erg} \mathrm{cm}^{-2} \mathrm{~s}^{-1}$ arcseconds ${ }^{-2}$, both in the red and in the blue! KCWI achieves this requirement by using a fixed (non-rotating) Nasmyth mount with a high-efficiency k-mirror de-rotator pre-focus, careful baffling and stray light design, and an optional nod and shuffle mode.

Flexibility and Operations Simplicity. The flexibility required for a general-purpose instrument is provided by multiple slicer formats, multiple gratings, and selectable grating tilts. We are planning a grating jukebox mechanism so that multiple gratings can be utilized on a single observing night without requiring nighttime grating changes, a necessity for WMKO operations.
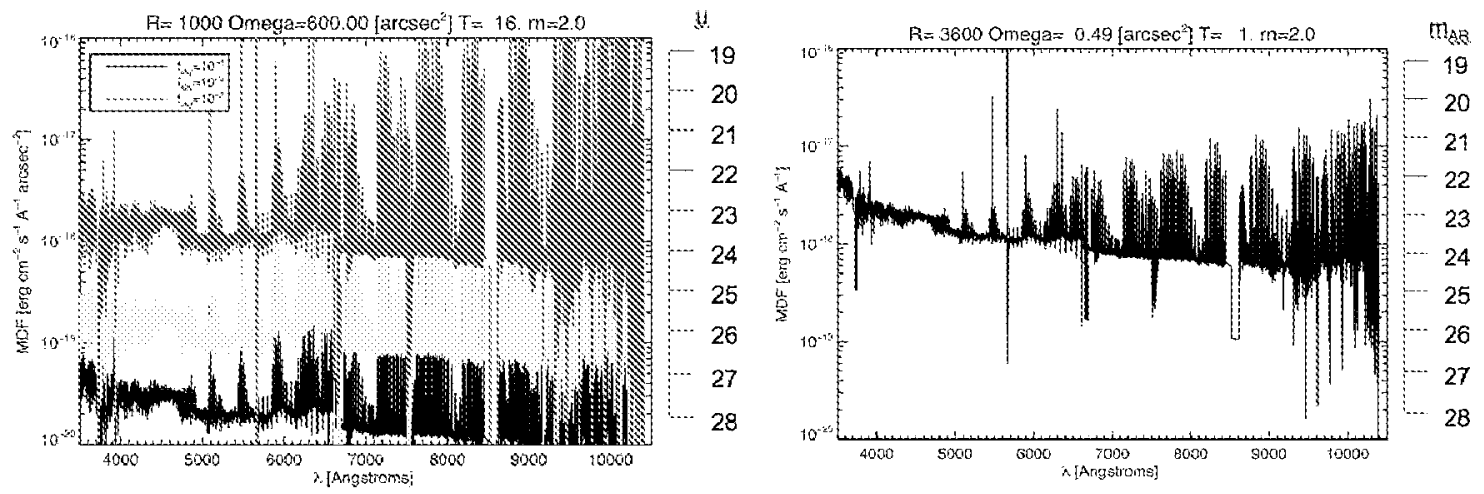

Figure 3: Left: sensitivity (at $\mathrm{S} / \mathrm{N}=\mathbf{1 0}$ per spectral resolution element) for "light-bucket" mode (binning entire IFU field-of-view) with a 16 hour integration at $\mathrm{R}=1000$ spectral resolution, and the coarse ( $30^{\prime \prime} \times 20$ ") IFU. Right: point source sensitivity, 1 hour exposure, $\mathrm{R}=3600$ (full band coverage). 
Table 1: KCWI science objectives and flowdown to requirements

\begin{tabular}{|c|c|c|c|c|c|c|}
\hline Science Objective & Spectral Content & $\begin{array}{l}\text { Spectral } \\
\text { Res }\end{array}$ & $\begin{array}{l}\text { Spatial } \\
\text { Res }\end{array}$ & Band & $\begin{array}{l}\text { Effic- } \\
\text { iency }\end{array}$ & FOV \\
\hline (1) Young stars Jets & $\mathrm{EmL}$ & HIG & $\mathrm{SL} *$ & $0870068 \mu \mathrm{m}$ & NED & Narow \\
\hline (2) Evolved stars/Nebulae & $\mathrm{EmL}, \mathrm{AbL}$ & MOD & Medium & 0.37 to $0.86 \mu \mathrm{m}$ & MED & Narrow \\
\hline (3) Light echoes & $\mathrm{SED}$ & MOD & SL-Coarse & $03710086 \mathrm{\mu m}$ & $M E D$ & Wide \\
\hline $\begin{array}{l}\text { (4) Star clusters-Kinematics, } \\
\text { Stellar pops, Abundances? }\end{array}$ & $\mathrm{AbL}$ & HIGH & $\mathrm{SL}$ & 0.37 to $0.86 \mu \mathrm{m}$ & MED & $\begin{array}{l}\text { Narrow- } \\
\text { Wide }\end{array}$ \\
\hline $\begin{array}{l}\text { (5) Nearby LSB, Burar galaxy } \\
\text { pops } 4 \text { kinematics, } \mathrm{H} \text { reglons }\end{array}$ & $\mathrm{EmL}, \mathrm{AbL}$ & $\begin{array}{l}\mathrm{MOD} \\
\mathrm{HCH}\end{array}$ & $\mathrm{SL}$ & 037 to 08 मn & $\mathrm{MED}$ & Wdes \\
\hline $\begin{array}{l}\text { (6) Galactic Halos (gas \& } \\
\text { stellar), Intracluster light }\end{array}$ & $\underset{\mathrm{z}}{\mathrm{EmL}, \mathrm{AbL} @ \text { low }}$ & $\begin{array}{l}\text { MOD- } \\
\text { HIGH }\end{array}$ & Coarse & 0.37 to $1.0 \mu \mathrm{m}$ & HIGH & Wide \\
\hline galaxies/nteractions low & $\mathrm{EnL}_{\mathrm{L}}, \mathrm{w} \mathrm{Hgh}$ & $\mathrm{MOD}$ & SL & $037010 \mathrm{um}$ & MED & Nanows \\
\hline (8) Circum QSO Medium & $\operatorname{EmL~} 2<\mathrm{z}<7$ & MOD & SL-Coarse & 0.37 to $1.0 \mu \mathrm{m}$ & MED & Wide \\
\hline 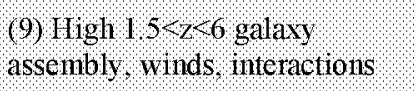 & $\mathrm{Bm}, \mathrm{Rest}$ Y & MOD & SL & 035 to $10 \mathrm{um}$ & HIGI & Warrows \\
\hline (10) Circum Galactic Medium & EmL, $2<z(L a)<7$ & MOD & SL/Med & 0.35 to $1.0 \mu \mathrm{m}$ & HIGH & Medium \\
\hline 11 y alpha enitters blobs & $\mathrm{EnL}_{5}, \mathrm{ra}$ ) & MOD & $\mathrm{SL} \mathrm{Med}$ & $01011 \mathrm{~m}$ & HIG & Wre \\
\hline (12) Cosmic Web Emission & EmL, $2<$ z(La) $<7$ & MOD & Coarse & 0.35 to $1.0 \mu \mathrm{m}$ & HIGH & Wide \\
\hline (13) Reionization 111 regions & $\mathrm{EnL}, 5<\mathrm{zLa})=$ & MOD & Coarse & $07 \mathrm{to} 1 \mathrm{\mu m}$ & HIGH & Wde \\
\hline
\end{tabular}

Table 2: KCWI science requirements

\begin{tabular}{|c|c|c|}
\hline Requirement & Baseline & Instrument Requirement \\
\hline SpatialResolution & 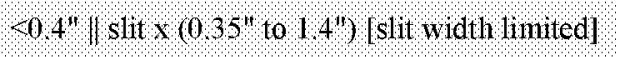 & \multirow{3}{*}{$\begin{array}{l}\text { multiple IUS, } \\
\text { slit limited optics, } \\
\text { (new blie camera) } \\
\text { multiple gratings }\end{array}$} \\
\hline 2. FOV & $2 \mathrm{D}: 20^{\prime \prime} \times(7 \text { to } 30)^{\prime \prime}$ & \\
\hline 3 spectral resolution & 1000 to 20000 & \\
\hline 4. Band & $\begin{array}{l}0.35 \text { to } 1 \mu \mathrm{m} \\
\text { simultaneous } \mathrm{H} \beta /[\mathrm{OIII}] \& \mathrm{H} \alpha /[\mathrm{SII}]\end{array}$ & $\begin{array}{l}2 \text { band-optimized channels } \\
\text { Blue: } 0.35 \text { to } 0.56 \mu \mathrm{m} \\
\text { Red: } 0.53 \text { to } 1 \mu \mathrm{m} \\
\text { Thick/depleted red CCD }\end{array}$ \\
\hline 5 efrensy & $\begin{array}{l}>025 \text { for faint objects/ow surface brighinesses } \\
\text { emission lines }\end{array}$ & V channels, nun gratings \\
\hline 6. Sky/object subtraction & $<0.01 \%$ sky & No flexure $\rightarrow$ K-mirror derotator; Nod \& shuffle \\
\hline 1 Object contrast & $10001[\mathrm{TBR}$ & Occuling spot? \\
\hline 8. Flexibility & High to accommodate a broad array of science & Multiple IFUs, multiple gratings, tilts \\
\hline 9 Operations & supple & Real-time reduction S W \\
\hline
\end{tabular}




\section{TECHNICAL DESCRIPTION}

\subsection{Overview}

Starting at the left hand side of Figure 4, the light from the telescope passes through the k-mirror image de-rotator and comes to a focus on the image slicer mirrors in the integral field unit (IFU). Light from the slicer mirrors is reflected back to the left and reaches a fixed pupil mirror array which has a center opening to allow the light from the telescope to reach the image slicer. The pupil mirrors form the entrance slit of the spectrograph as well as a common pupil for the system, and the light from the pupil mirrors proceeds to the left again, reaching the collimator mirror at the upper right center in Figure 4. After the collimator the light is split into two beams (red and blue) by a dichroic. The optical paths of the instrument's red and blue channels are composed of the same elements. Each has a fold mirror, a transmission VPH grating, a spectrograph camera, and the detector. The grating is mounted on a rotation stage for angle adjustment. The camera and detector are mounted on a polar motion stage that can swing the camera and detector through a range of dispersion from the grating to allow selection of the desired bandpass and maximize efficiency for the desired bandpass. KCWI will have multiple gratings, and the preferred configuration is to provide an automated system for interchanging the gratings.

The blue channel of KCWI will cover the wavelength range from 0.35 to $0.56 \mu \mathrm{m}$, and the red channel will cover the wavelength range from 0.53 to $1 \mu \mathrm{m}$. The candidate sampling scales for the KCWI IFU are $0.35 ", 0.7$ ", and 1.4 " slice widths x 24 slices, giving fields of view of $20 "$ × 8.4", 20" x 16.8", and 20" x 33.6". The cameras will use detectors with $15 \mu \mathrm{m}$ pixels providing $\sim 4$ pixel sampling over a $0.6 "$ seeing disk in the spatial direction. The red camera will use a $4 \mathrm{k} \mathrm{x} 4 \mathrm{k}$ red optimized thick substrate CCD, and the blue camera will use an e2v technologies 231-84 4k x 4k $15 \mu \mathrm{m}$ pixel back illuminated, thinned CCD with a broad band AR coating. A selection of $\sim 12 \mathrm{VPH}$ gratings will allow spectral resolutions from 1,000 to 20,000 with excellent sensitivity. The total throughput including the telescope and atmosphere is predicted to be at least $20 \%$ over the full wavelength range $(0.35 \mu \mathrm{m}$ to $1 \mu \mathrm{m})$ with a mid-band peak of $35 \%$.

KCWI will also benefit from the heritage of previous WMKO instruments for its electronics and control software. Appropriate heritage from the recent LRIS red upgrade ${ }^{[7]}$ will be used for the red channel detector and read out system and the supervisory controls and motion control will be a combination of CWI heritage and WMKO standard electronics designs. Software will be based on the WMKO standard client-server architecture with DS9 used for image display. A custom data reduction pipeline for the IFS will be developed using IDL. The KCWI team and the Observatory hope to fast track the development of KCWI with delivery of KCWI to the Keck II telescope in late 2012.

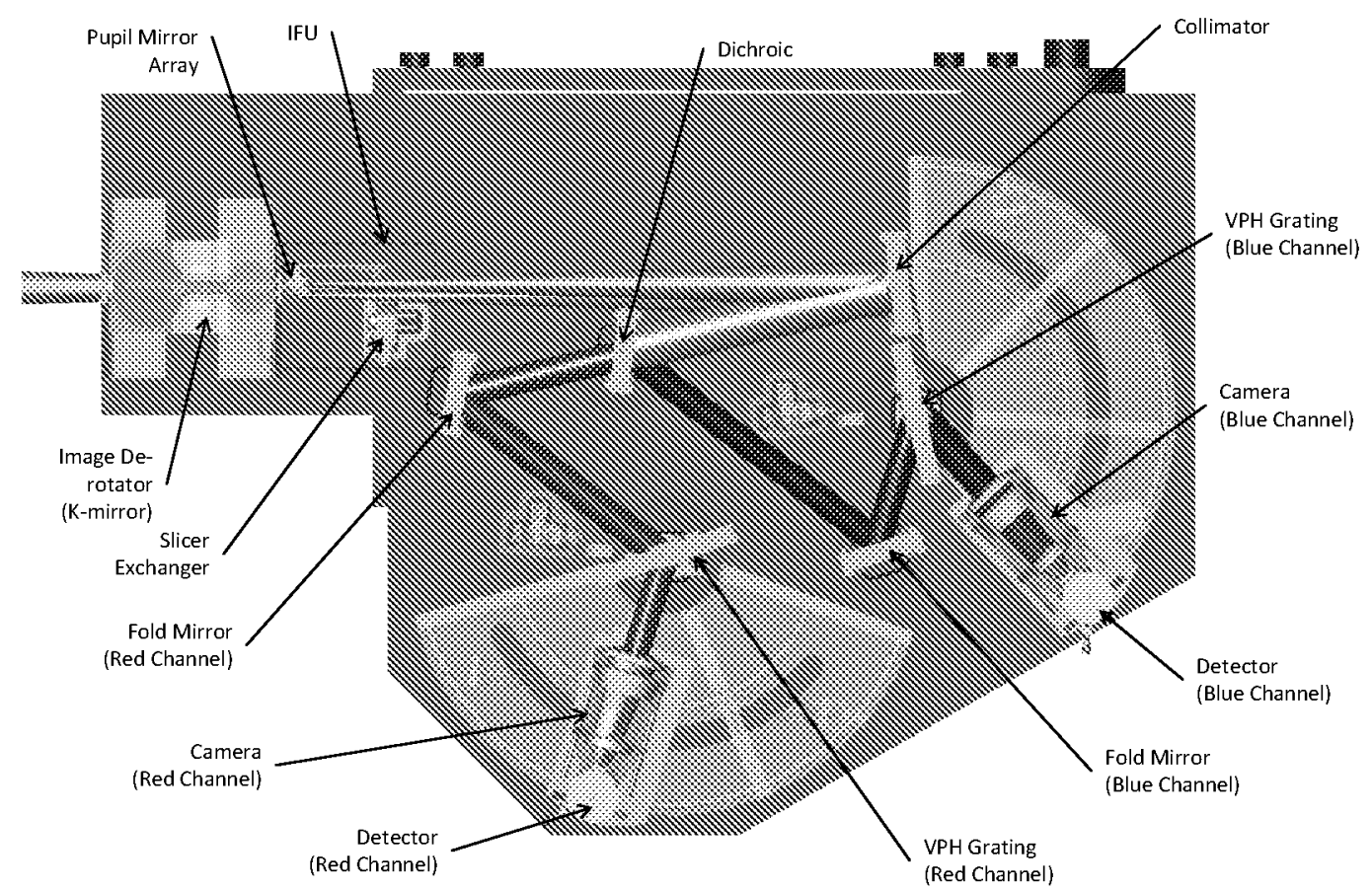

Figure 4: KCWI plan view optical layout 


\subsection{Optics}

Image De-rotator. The KCWI image de-rotator is a build-to-print copy of the Keck AO de-rotator. It is a classical Kmirror de-rotator with a $2^{\prime}$ field of view. The three mirrors will be coated with an advanced protected silver plus multilayer coating developed at UCO, which leads to at most a $10 \%$ loss in efficiency $\left(\mathrm{R}_{\mathrm{m}}{ }^{3}>0.9\right)$ while vastly simplifying the mechanical design, and essentially eliminating flexure and the associated compensation mechanisms and software.

Guider. The KCWI guider will be based on the MAGIQ guider currently implemented on several instruments at $\mathrm{WMKO}^{[8],[9]}$. The guider will have a baseline $2^{\prime} \mathrm{FOV}$. The details of the guider optical feed will be addressed in the preliminary design however a possible solution is to have an annular fold mirror surrounding the IFU slicer stack. The fold mirror is be angled such that the annular field is directed to the guider. The MAGIQ guider incorporates a low order wavefront sensing system that will also be used in KCWI for control of the telescope focus.

IFU. KCWI uses a reflective image slicer rather than a fiber IFU. A slicer provides four important advantages: high throughput in the blue, avoids the packing inefficiency in the gaps between spectra and between fibers, no transmission variability due to flexure that would degrade sky subtraction accuracy, and an easily implemented selection of formats. A small disadvantage is defocus at the ends of the slices due to tilt, and this defocus is included in the total imaging error budget. KCWI will provide three image slicer formats, each with a different slice width, a fixed slice length and 24 slices. The slicers are mounted on a linear slide and can be exchanged in less than a minute. (Note that this works in the optical where the detector pixels can be binned for the wider slice IFUs, and the read noise is low). The multiple slicers feed a fixed pupil mirror array, so that the resulting virtual slit is very similar in the three cases except for slit width. The FOV is then $20^{\prime \prime} \times \Delta \phi$, where $\Delta \phi=24 \theta$. A candidate selection is $0.35^{\prime \prime}, 0.7 "$, and $1.4 "$ slice widths $\times 24$ slices, giving fields of view of $20 "$ x 8.4", 20" x 16.8", and 20" x 33.6". The CCD has $15 \mu \mathrm{m}$ pixels which oversample all IFU formats, and provides $\sim 4$ pixels per $0.6^{\prime \prime}$ seeing disk in the spatial direction (binning is possible to reduce read noise). The CWI IFU, shown in Figure 5, is diamond turned out of aluminum and coated. For KCWI the requirements for surface quality and edge filling factor are tighter, particular for the finest slicer, and we will use a proven Zerodur glass slicer technique built by Winlight, Inc. in Marseille, France. A simple bright source occulting spot can be placed just in front of the slicer when desired for high dynamic range observations, using a slide or pivot mechanism.

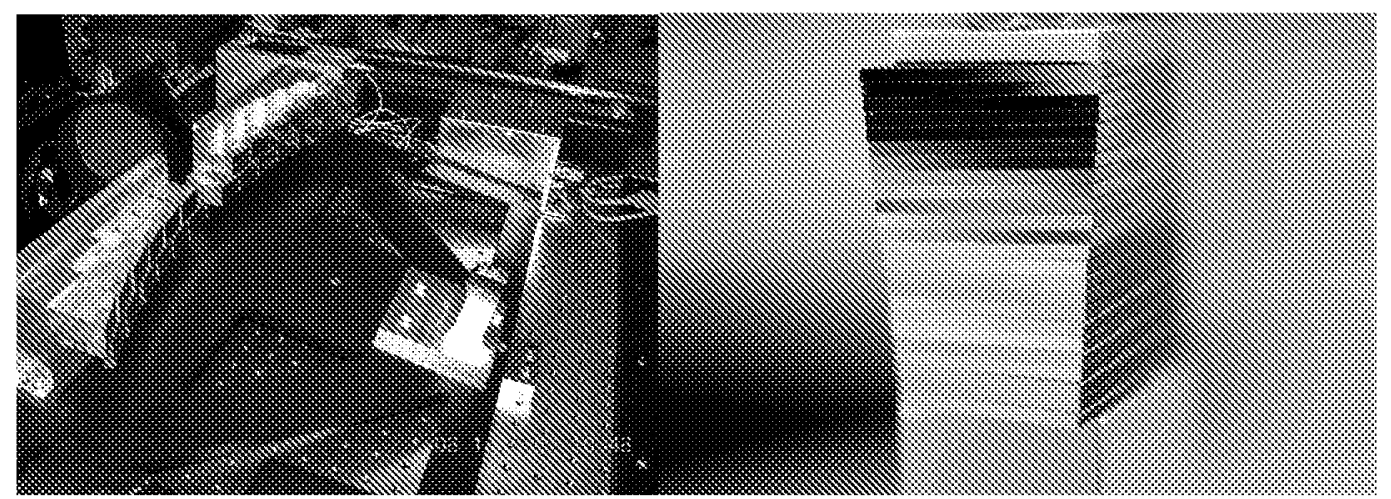

Figure 5: CWI IFU (left) and image slicer (right), all aluminum, diamond turned mirrors. The KCWI slicer will be polished Zerodur.

Collimator. The KCWI collimator is spherical, has moderate figure requirements, and is mounted on a translation stage to provide internal slicer to detector focus adjustment.

VPH gratings for high transmission. KCWI will use VPH gratings as adopted by CWI, which provide very high efficiency when operated near the Bragg condition. The grating and camera/CCD dewar assembly are each mounted on a rotating articulation unit that provides an arbitrary incidence angle to the grating $(\alpha)$ and exit angle to the camera/detector $(\beta)$. These angles are typically similar, and relatively low for low dispersion gratings, but can be as high as $60^{\circ}$ for a high dispersion grating. The grating curves and articulation arrangement are illustrated in Figure 6 for the CWI grating. The angles can be changed in a matter of a minute to adjust the instantaneous bandpass over the "available" bandpass. The available bandpass for a given grating is determine by the range of simultaneously high s- and p-polarization efficiencies. Gratings will be changed either manually, or with a "jukebox" mechanism. The latter will be studied and costed during the preliminary design. 
Filter Wheels. Stray out of band light can be reduced to negligible levels by interposing a band-limiting filter. The filter will be located between the grating and the camera. A complement of 6 per channel mounted on a filter wheel or a slide mechanism, or in some cases fixed to each grating will provide complete flexibility.
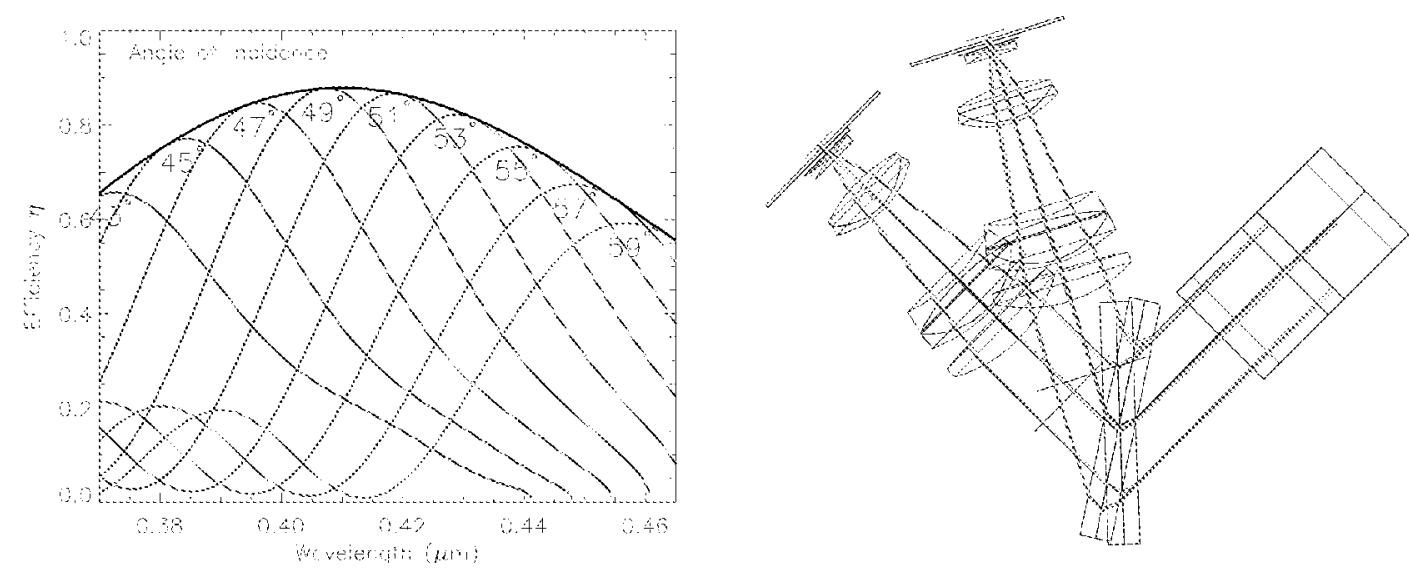

Figure 6: Left: the red curves show response vs. wavelength at a single grating/camera angle for high-dispersion grating, and the black curve shows the "superblaze" envelope of peak efficiencies at different angles. Right: schematic showing two grating/camera$\mathrm{CCD}$ angles for the CWI high-dispersion grating.

Cameras. Both red and blue cameras are designed to be simple to fabricate and provide excellent transmission. Both have $\sim 150 \mathrm{~mm}$ pupils and $300 \mathrm{~mm}$ focal lengths. The blue camera uses 9 all-spherical lens elements (including fieldflattener and vacuum lens) and blue optical "i-line" glasses formulated for high transmission in the UV. No exotic materials or other new technologies are required to obtain excellent transmission and imaging performance over the 0.34 to $0.58 \mu \mathrm{m}$ range. The red camera uses 8 all-spherical lens elements and obtains $<1$ pixel $(15 \mu \mathrm{m})$ rms image diameters. Both cameras will achieve greater than $75 \%$ transmission. Because of the use of simple surfaces and materials both cameras are likely to achieve theoretical performance at a reasonable cost. Figure 7 shows the blue camera conceptual design and imaging performance.
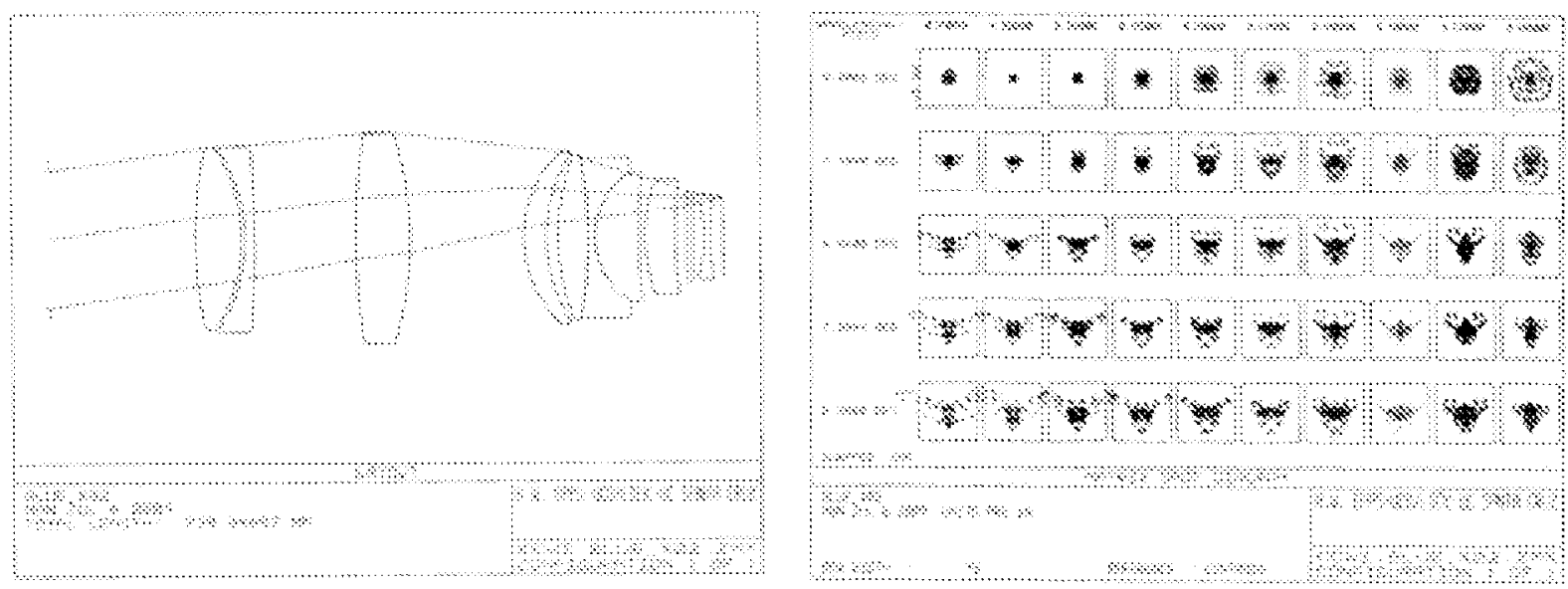

Figure 7: Blue camera performance (right) and early concept lens design (left)

Detectors. The blue CCD is a production $\mathrm{e} 2 \mathrm{v} 231-844 \mathrm{k}$ x $4 \mathrm{k} 15 \mu \mathrm{m}$ pixel back-thinned, AR coated device that routinely achieves $>80 \%$ QE and 2 to $3 \mathrm{e}$ - (rms) read noise. The red CCD will be a LBNL fully-depleted $4 \mathrm{k} \times 4 \mathrm{k} 15 \mu \mathrm{m}$ pixel p-channel device. Typical QEs are $90 \%$ peak and $60 \%$ at $1.0 \mu \mathrm{m}$. These devices have low charge diffusion, good MTF, and zero fringing. 
Calibration. KCWI includes a calibration system based on CWI that simulates the telescope input beam and provides continuum (LEDs) and line sources with diffuse illumination and a calibration grid. Calibration light is introduced by inserting a pick-off flat into the input beam path.

CWI Status. CWI has just completed its second engineering run at Palomar. Spectral and spatial resolution requirements were verified. Photographs of the instrument are shown in Figure 8 and a spectrum of the Ring Nebula in Figure 9.

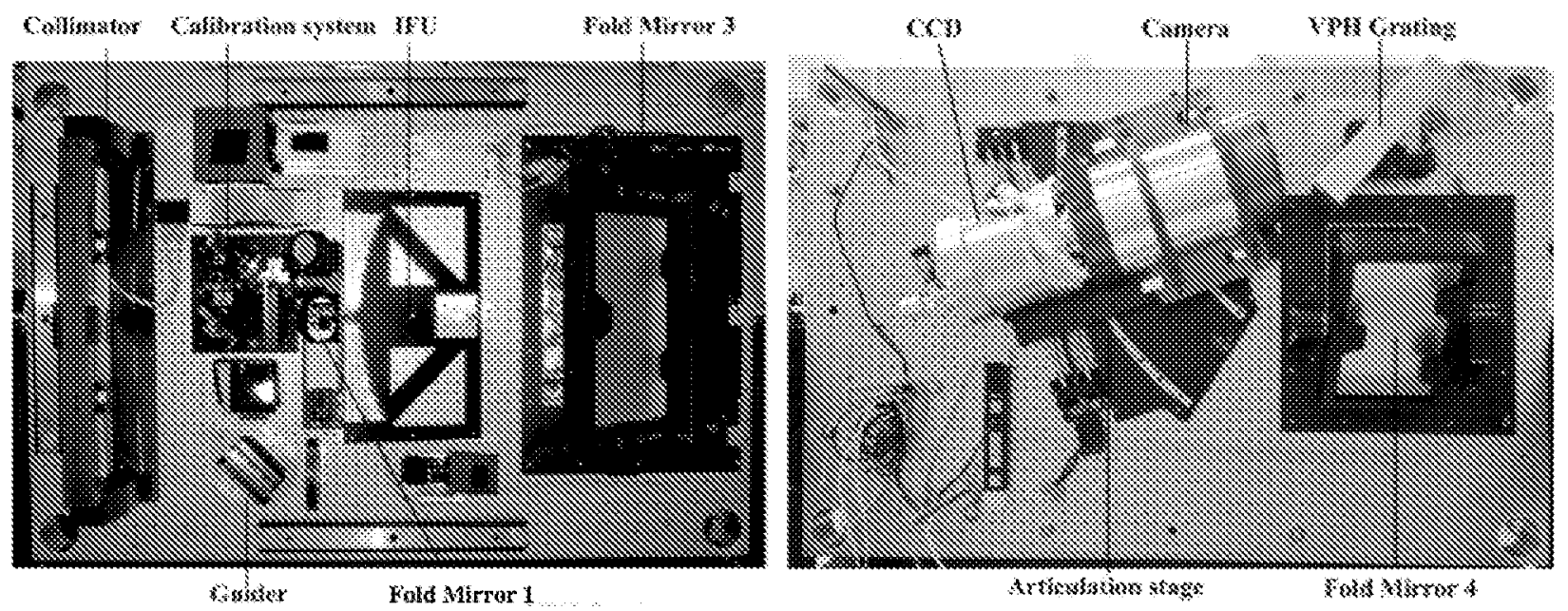

Figure 8: CWI - top and bottom of breadboard.

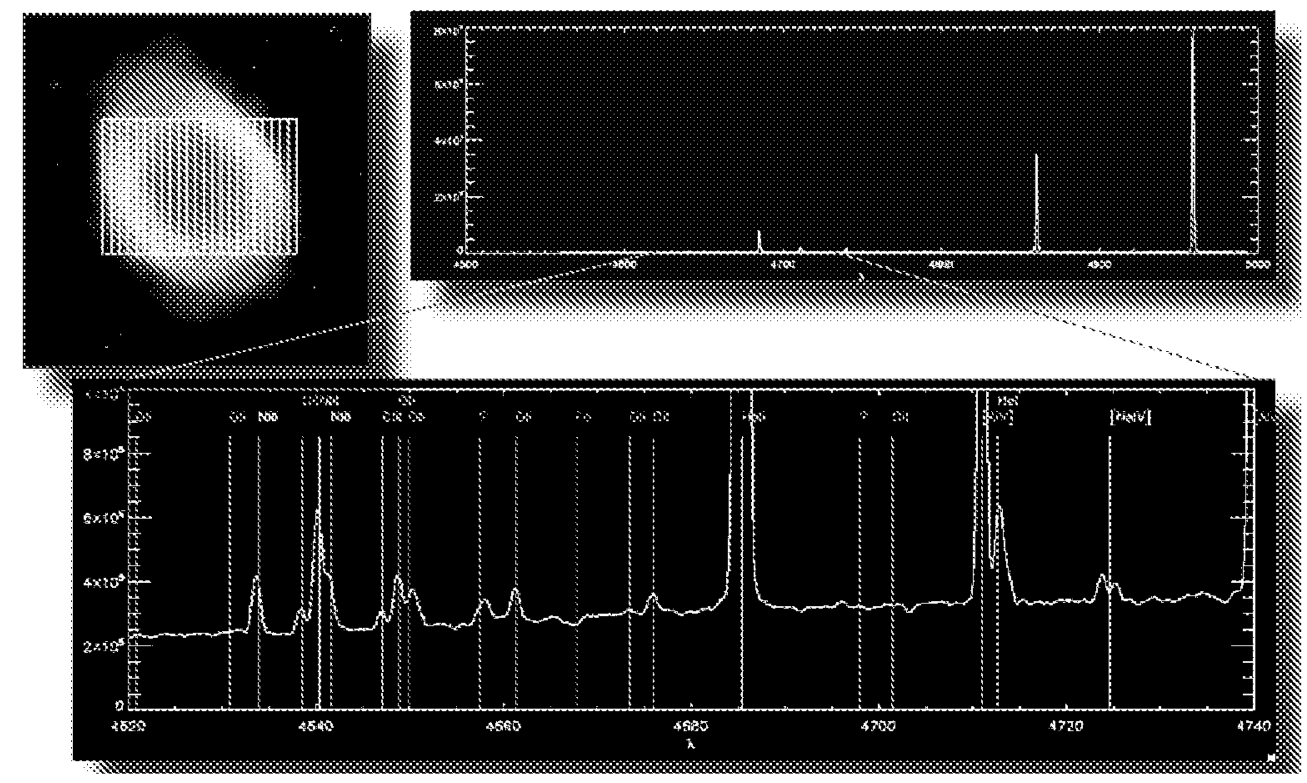

Figure 9: 10 minute CWI spectrum of Ring Nebula showing weak lines and $\mathrm{R} \sim 5000$ resolving power

Required Technical Developments. We anticipate that the principle technical developments are the large, high dispersion VPH gratings for the highest resolution modes, and the image slicer. All but one of the KCWI unique gratings are less challenging than the CWI blue grating. We plan to obtain prototypes of several of the more challenging KCWI gratings during the preliminary design (PD) phase. The finer slicer proposed for KCWI will push the limits of slicer fabrication, but not inordinately. Some prototyping will be performed during PD. The LBNL fully depleted CCDs are 
transitioning from developmental to more routine, but we plan to obtain a science grade CCD during the PD phase to lower risk.

\subsection{Mechanics}

The use of a de-rotator makes the KCWI mechanical design very simple and robust, and makes the instrument easy to implement, operate, and maintain over time. As shown in Figure 4, all optical elements are mounted on a single highstiffness custom Newport optical breadboard in a single, horizontal plane. The breadboard will be enclosed to protect the optical system from dust and will be mounted on a standard WMKO handling cart and rail system with the electronics mounted below the breadboard in glycol cooled enclosures. During observing KCWI will be located at the Keck II right Nasmyth focus on the telescope's Nasmyth platform. The optical mounts, collimator focus stage, grating rotation stage, and camera/CCD articulation stage will be adaptations of proven CWI designs, built by Newmark Systems, Inc., and will be made simpler and more robust by the fixed KCWI orientation.

\subsection{Sensitivity and Sky Subtraction}

Transmission and Sensitivity. Based on the use of high efficiency VPH gratings and detectors we estimate that the instrument+telescope+atmosphere end-to-end throughput will exceed $20 \%$ as shown in Figure 10 and may peak as high as $35 \%$. Instrument overhead should not differ significantly from other WMKO instruments such as ESI that do not use slit masks.

Nod and Shuffle (N\&S). This mode provides excellent sky subtraction by ensuring that "source" and "background" spectra are imaged by identical optical elements and detector pixels. ${ }^{[10]} \mathrm{N} \& \mathrm{~S}$ will be implemented to ensure excellent sky subtraction. There are two possible modes. In mode 1 a retractable mask blocks $\sim 1 / 2$ of the IFU to create a storage area. A source exposure is obtained $(\sim 1 \mathrm{~min})$, the shutter is closed, the charge on the CCD is shifted by 66 pixels $\left(10^{\prime \prime}\right)$, the telescope is nodded by $>30$ ", and a "background" spectrum is obtained (same exposure). This is followed by an opposite charge shift on the CCD shift and telescope motion to return to the source. In mode 2 the full IFU field is available with reduced spectral coverage. The CCD is rotated 90 degrees and a retractable mask blocks the upper and lower third of the CCD (in the spectral direction). N\&S proceeds identically now with a 1666 pixel shift to alternately place "source" and "background" in the masked storage areas. Mode 2 is used on CWI
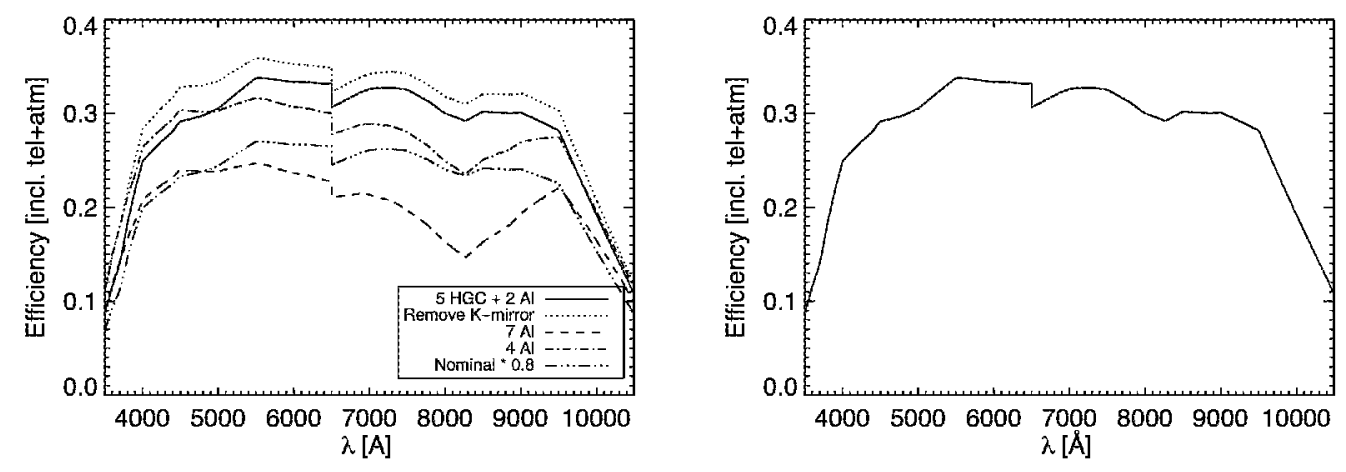

Figure 10: Left: improved efficiency using different mirror coatings (shown with out including the de-rotator). Right: net efficiency (instrument + telescope + atmosphere).

\subsection{Instrument Electronics and Software}

The KCWI electronics and software will be based on components used in recent WMKO instruments in addition to the recently commissioned CWI instrument. This will reduce development time and also ensure long term maintainability after the instrument is commissioned. The detector readout systems will be Astronomical Research Cameras, Inc. (ARC) CCD controllers that are used in all of the current visible wavelength instruments at WMKO, including the recent upgrade to the LRIS red channel ${ }^{[7]}$. The LBNL devices require a high voltage substrate bias, and we plan to base the KCWI red CCD readout system on the system developed for the LRIS upgrade. The blue channel readout system will be a more standard configuration, based on the blue channel of the LRIS instrument. In both cases pre-amplifiers for the CCD video outputs will be located at the dewars. The ARC CCD controllers will also provide temperature monitoring and control for the detectors. 
The motion control systems for selection of the IFU slicer scale, and the grating and camera articulation and several other active mounts will be supplied by Newmark Systems, Inc. The motion controller for the instrument hatch, Kmirror, filter wheels, and the grating changer will be Danaher Motion single axis controllers very similar to those used in the MOSFIRE instrument. The motion controllers will be interfaced to a terminal server which will in turn communicate with the instrument host computer via Ethernet. Power control for the instrument will be provided by two Eaton (Pulizzi) IPC3401-NET Ethernet controlled power distribution unit. The Eaton power controllers will also be used to select the calibration light sources.

The KCWI instrument software will be based on components developed for the LRIS red upgrade and for the MOSFIRE instrument. The new LRIS red channel CCD read out and target software should be relatively straightforward to port to the KCWI 4k x 4k LBNL detector. The blue channel CCD read out software can also be based on this core platform. The power controller and the Danaher Motion controller software will be a straightforward port from MOSFIRE. The low level and global server architecture will also be re-used from MOSFIRE. The only new software that will need to be developed will be the low level server for the Newmark motion controllers, and this will be based on the software developed for CWI. The instrument graphical user interface (GUI) designs are to be determined, but they could easily be based on a port of the MOSFIRE desktop and control GUIs.

\section{ACKNOWLEDGEMENTS}

The W. M. Keck Observatory is operated as a scientific partnership among the California Institute of Technology, the University of California, and the National Aeronautics and Space Administration. The Observatory was made possible by the generous financial support of the W. M. Keck Foundation. This material is based in part upon work supported by AURA through the National Science Foundation under Scientific Program Order No. 5 as issued for support of the Telescope Systems Instrumentation Program (TSIP), in accordance with Proposal No. AST-0335461 submitted by AURA.

\section{REFERENCES}

[1] Chang, D. Y., Martin, D. C., Matuszewski, M., Morrissey, P., Moore, A. M., Rahman, S., \& Crabill, R. "The cosmic web imager integral field spectrograph design and first results", In Proceedings of the SPIE 7735, 25. (2010).

[2] Neill, J. D., Martin, C., \& Seibert, M. "The mass loss history of Mira", Bulletin of the American Astronomical Society 39, p. 855. (2009).

[3] Wareing, C. J., Zijlstra, A. A., O'Brien, T. J., \& Seibert, M. "It's a wonderful tail: The mass loss history of Mira", Astrophysical Journal Letters, accepted. (2009).

[4] Noyola, E., Gebhardt, K., \& Bergmann, M. "Central dynamics of globular clusters: the case for a black hole in $\omega$ Centauri", In Proceedings of the International Astronomical Union 246, 341-345. (2008).

[5] Cen, R. "HI Cosmology: Expectations at $\mathrm{z}>6$ ", In AIP Conference Proceedings 1035, 10-16. (2008).

[6] Cantalupo, S., Porciani, C., \& Lilly, S. J. "Mapping neutral hydrogen during reionization with the Lya emission from quasar ionization fronts", The Astrophysical Journal 672(1), 48-58. (2008).

[7] Rockosi, C. M., Stover, R., Kibrick, R. I., Lockwood, C., Peck, M., Cowley, D. J., Bolte, M., Adkins, S. M., Alcott, B., Allen, S. L., Brown, W. F., Cabak, G. F., Deich, W. T. S., Hilyard, D. F., Kassis, M. F., Lanclos, K., Lewis, J. P., Pfister, T., Phillips, A. C., Robinson, L., Saylor, M., Thompson, M., Ward, J., Wei, M., \& Wright, C. A. "The low-resolution imaging spectrograph red channel CCD upgrade: Fully depleted, high-resistivity CCDs for Keck", In Proceedings of the SPIE 7735, 27. (2010).

[8] Adkins, S. M., Cohen, J., Aycock, J., Bell, J., Cohen, R., Cooper, A., Goodrich, B., Johnson, J., Kwok, S. H., Lyke, J., Neyman, C., Nordin, T., Panteleev, S., Tolleth, G., \& Tsubota, M. "MAGIQ at the W. M. Keck Observatory, initial deployment of a new acquisition, guiding and image quality monitoring system", In Proceedings of the SPIE 7014, 70141U. (2008).

[9] Kwok, S. H., Johnson, J., Adkins, S. M., \& McCann, K. "The software for MAGIQ: a new acquisition, guiding and image quality monitoring system at the W. M. Keck Observatory", In Proceedings of the SPIE, 7019, 10. (2008).

[10] Sembach, K. R., \& Tonry, J. L., "Accurate Sky-Subtraction of Long-Slit Spectra: Velocity Dispersions at Sigma_V=24 Mag/arcsec"," Astron. J., 112, 797 (1996). 